\title{
Mistura de biodiesel de sebo bovino em motor diesel durante 600 horas
}

\author{
Blend of biodiesel from beef tallow in a diesel engine during 600 hours of tests
}

\author{
Ila Maria Corrêa ${ }^{\mathrm{I}}$ José Valdemar Gonzalez Maziero $^{\mathrm{I}}$ Moisés Storino $^{\mathrm{I}}$
}

RESUMO

O biodiesel de sebo bovino é considerado uma alternativa de baixo custo e de grande disponibilidade por ser resíduo da produção agropecuária brasileira, que é uma das maiores do mundo. Raros são os trabalhos que mostram a utilização do biodiesel de sebo bovino em motores diesel. Assim, o objetivo deste trabalho foi verificar o efeito da mistura de biodiesel bovino na proporção de 5\% ao óleo diesel comercial no desempenho do motor, possíveis consequências internas no motor e nas características do óleo lubrificante após o uso prolongado em motor diesel. Foram realizados ensaios em bancada dinamométrica utilizando um trator agrícola. O desempenho do motor foi determinado através da tomada de potência (TDP). O motor foi operado por $600 \mathrm{~h}$ durante as quais foi determinada a potência, o consumo de combustivel $e$ analisadas as amostras de óleo lubrificante a cada 100h. Ao final do ensaio, o motor foi aberto e inspecionado. A análise do óleo lubrificante mostrou nível de contaminação crítico a partir das 400h, mas a inspeção visual do motor não detectou nenhum desgaste interno. O motor funcionou normalmente, embora tenha ocorrido tendência de redução na potência e aumento de consumo de combustível ao longo das 600h.

Palavras-chave: biocombustível, energia, desempenho de motor.

\section{ABSTRACT}

Biodiesel from beef tallow has been considered a low-cost and high availability alternative due to be residue from the Brazilian livestock production, one of the world's largest. Papers that show the use of biodiesel from beef tallow in diesel engine are rare. The aim of this study was to investigate the effect of blend of biodiesel from beef tallow (B5) in commercial diesel oil on engine performance, analyzing possible internal consequences and characteristics of lubricating oil after the prolonged use in a diesel engine. Engine performance was evaluated through tractor power take off (PTO) tests. The engine was operated for 600 hours. Power and fuel consumption were measured. Samples of lubricating oil were analyzed by the end of each 100 partial hours. At the end of the test the engine was opened and visually inspected. Analysis of engine lubricating oil has showed critical level of contamination after 400 hours but the visual inspection of engine did not detect any internal wear. The engine performance was satisfactory although there was tendency of reduction in power and increase on fuel consumption along the 600 hours.

Key words: biocombustible, energy, engine performance.

\section{INTRODUÇÃO}

O sebo bovinoé uma das principais matérias primas para a produção de biodiesel, sendo superado apenas pelo óleo de soja. Isso é favorecido pelo fato de o sebo ser resultante do abate de gado para produção de carne produzida no Brasil, que é um dos maiores produtores do mundo, tendo, portanto, baixo custo de comercialização (MANSINI et al., 2007). Conforme BELLAVER (2008), além do volume aproveitado na indústria de cosméticos e no processo de fabricação de borracha, ainda sobram 500 mil toneladas anuais de matéria prima, que pode constituir um problema ambiental.

Uma vantagem do biodiesel de sebo bovino apontada por MILLER KLEIN (2006) é o número de

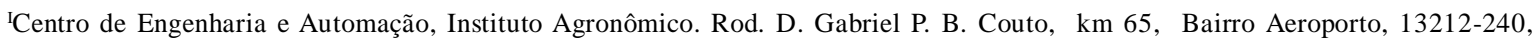
Jundiaí, SP, Brasil. E-mail: imcorrea @iac.sp.gov.br. *Autor para correspondência. 
cetano maior que o do óleo vegetal, o que permite combustão mais eficiente em motores diesel. Destaca, entretanto, como desvantagem o alto ponto de névoa, pois, devido ao elevado nível de gorduras saturadas, tende a cristalizar as temperaturas muito mais altas do que o biodiesel de óleo vegetal.

Os trabalhos científicos encontrados sobre biodiesel de sebo bovino abordam em geral a sua avaliação físico-química (MOURA et al., 2007; MANSINI et al., 2007, BETTO \& PRADO, 2008) e não sua aplicação em motor. Nesse sentido, foram encontrados apenas os trabalhos de GARCIA \& TOOKUNI (2006) e de MAZIERO et al. (2009). Daí o interesse em avaliar o desempenho de um motor diesel utilizando biodiesel de sebo bovino em mistura ao óleo diesel comercial em ensaios dinamométricos.

É possível, porém, encontrar trabalhos sobre o desempenho mecânico do motor com o uso de biodiesel feito a partir de outras matérias-primas, sendo que boa parte desses trabalhos foca a realização de testes de curta duração, nos quais são levantados dados sobre o desempenho (torque, potência, consumo de combustível). Desses testes, somente se pode inferir se o motor alimentado com biodiesel fornece nível de potência similar ou diferente ao obtido com óleo diesel. Já nos ensaios de longa duração, é possível observar, além da potência e consumo de combustível, os efeitos do biodiesel sobre desgaste, durabilidade e inspeção interna dos componentes dos motores. Para SILVA (2006), a complexidade e o custo dos ensaios de longa duração como o de durabilidade tornam escassas as publicações de tais resultados.

Alguns dos trabalhos cuja abordagem é o uso prolongado de biodiesel são relatados a seguir. Destaque-se que não há uniformidade sobre o número de horas desejáveis para caracterizar um ensaio de "longa duração". SALVADOR et al. (1986) testaram durante 30 horas um trator Agrale 4100 acoplado a dinamômetro com óleo diesel e três misturas (B33, B67 e B100) de éster metílico de boleira (Joannesia princeps, Vell). Não foram identificadas irregularidades no funcionamento do motor durante os testes, mas houve formação de uma fina camada de resíduos de carbono na cabeça do pistão e adjacências e sem aparente corrosão ou depósitos no bico injetor.

O efeito em longo prazo de uma mistura de óleo diesel (80\%) e biodiesel (20\%) foi estudado por ALI \& HANNA (1996) em relação ao desempenho e desgaste de um motor diesel. Constataram que o motor operou satisfatoriamente por $148 \mathrm{~h}$, tendo a potência, o torque e o consumo específico de combustível se mantido constantes. A análise do óleo lubrificante, a intervalos de $45 \mathrm{~h}$, mostrou que a redução na viscosidade após $100 \mathrm{~h}$ de operação foi típica de uma operação normal com óleo diesel. O desgaste do motor avaliado, com base na concentração de metais no óleo lubrificante, permaneceu dentro do limite normal recomendado para aquele tipo de motor.

Testes com 1000h de duração realizados por PETERSON et al. (1999) mostraram que o motor, quando alimentado com B100 (éster etílico de óleo de soja hidrogenada), desenvolveu menos potência que as misturas B50 e B25. Por outro lado, o consumo de combustível com B100 nem sempre foi maior do que o apresentado pelas misturas ao longo do ensaio.

A utilização de óleo de soja degomado misturado ao diesel comercial B2 nas proporções de 0,5 e $50 \%$ foi avaliada por INOUE et al. (2009) em ensaio de 300 horas de funcionamento de um grupo gerador composto por motor Agrale M90 (96kW a 2500rpm). Após aberto, foi encontrada em todos os tratamentos deposição de resíduos no cabeçote do motor e na válvula de admissão, com intensidade maior na mistura de $50 \%$. Não foram verificados desgastes dos componentes do motor.

O óleo lubrificante é responsável pela redução do atrito e consequentemente desgaste do motor, bem como pela limpeza dos resíduos da combustão e dos resíduos provocados pelo desgaste dos componentes do motor. A eficiência do lubrificante pode ser diminuída pela utilização severa do motor e por contaminantes, tais como, água, poeira (sílica), combustíveis e resíduos da combustão. Através da análise periódica do lubrificante, é possível acompanhar a sua deterioração normal e aquelas causadas por contaminações diversas, servindo como indicativo de manutenções preventivas. A análise do teor de contaminação do óleo lubrificante, segundo PETERSON et al. (1999), fornece uma excelente indicação das condições internas do motor. Os autores analisaram a contaminação do óleo lubrificante por metais (alumínio e ferro) em um motor diesel, a intervalos de $100 \mathrm{~h}$, durante o ensaio de 1000, com misturas (B25, B50 e B100) de éster etílico de óleo de soja hidrogenada. Sem referir se os valores encontrados estão dentro da faixa de normalidade, concluíram apenas que a presença dos metais com o uso de B100 foi igual ou menor que a ocorrida com B25 ou B50. Nos testes de durabilidade realizados por KAUFMAN \& ZIEJEWSKI (1984), com éster metílico de óleo de girassol, o consumo de óleo lubrificante foi satisfatório, sendo observada diluição de combustível no óleo lubrificante.

Um motor diesel foi submetido a ensaios por CORRÊA et al. (2008), a um regime de cerca de 100 horas de funcionamento sob diferentes cargas, com B100 de girassol. A análise do óleo lubrificante antes e 
após o uso com B100 detectou alterações aceitáveis, sendo a viscosidade, a presença de água e o teor de ferro as variáveis mais expressivamente alteradas.

Já no estudo realizado por INOUE et al. (2009), a análise do óleo lubrificante indicou valores críticos, sugerindo a sua substituição a intervalos reduzidos inferiores as $150 \mathrm{~h}$ preconizadas pelo fabricante.

Assim, o objetivo deste trabalho foi verificar o efeito da mistura de biodiesel bovino na proporção de 5\% ao óleo diesel comercial no desempenho do motor, e as possíveis consequências internas nos elementos e nas características do óleo lubrificante após o uso prolongado.

\section{MATERIAL E MÉTODOS}

O biocombustível adquirido para este trabalho era composto de $70 \%$ de biodiesel de sebo bovino e $30 \%$ de biodiesel de algodão e foi obtido mediante rota metílica pela empresa SP BIO Ind. e Com. de Biodiesel Ltda. Essa composição se deve, provavelmente, pelo fato de a matéria prima principal necessitar, às vezes, de outra para alcançar as especificações exigidas para o biodiesel comercializado no Brasil. As características físico-químicas foram apresentadas pelo fornecedor e também determinadas em Laboratório do Instituto Tecnológico de Pesquisas, sendo comparadas ao especificado pela Resolução ANP n.7/2008 que estabelece a especificação do biodiesel comercializado no país.

Os ensaios foram realizados em bancada dinamométrica utilizando-se um trator Valmet 68 , ano 1983, equipado com motor MWM D229.3 novo, com apenas 50h de amaciamento. Para os ensaios, o trator foi acoplado ao dinamômetro Schenck W 400 por meio da sua tomada de potência. Os parâmetros de desempenho determinados foram: rotação do motor em rpm, rotação da tomada de potência (TDP) em rpm, torque na TDP em Nm; consumo horário em $\mathrm{L} \mathrm{h}^{-1}$; e consumo específico em $\mathrm{g} \mathrm{kWh}^{-1}$. As rotações foram obtidas por meio de pick-up magnético e roda dentada, o torque era fornecido pelo dinamômetro, o consumo horário foi obtido por meio de um medidor de vazão volumétrico Micro Oval II modelo LS4150 e o consumo específico foi calculado em função da densidade do combustível. Por meio de sensores eletrônicos, foram monitoradas as temperaturas nos seguintes elementos: óleo lubrificante, combustível, ambiente, saída de água do motor, ar de admissão e ar da tubulação de escapamento. Os dados foram coletados utilizando-se um aquisitor de dados (datalogger) marca Campbell. Um programa de aquisição de dados foi desenvolvido para utilização e processamento via Excel. Nesse ensaio, o motor funcionou durante $600 \mathrm{~h}$ apenas com a mistura B5 de sebo bovino adicionado ao óleo diesel comercial B3. De certa forma, poder-se-ia dizer que a mistura gerada seria um B8, mas como a principal matéria prima estudada era o biodiesel de sebo bovino, considerouse adequado designá-lo dessa forma.

Para avaliação de efeitos de longo prazo, o motor foi submetido a diversos ciclos de carga, utilizando somente a mistura estudada, seguindo procedimento da EMA - Engine Manufacturer's Association, adotado por PETERSON et al. (1999). Este ciclo de carga simula diferentes condições de funcionamento do motor de forma intensiva. Ao longo do dia, foi possível executar três ciclos de carga totalizando nove horas diárias. Amostras de óleo lubrificante foram tomadas para análise a cada $100 \mathrm{~h}$ de funcionamento do motor, visando verificar possível contaminação pelo combustível com os laudos de análise realizados pela empresa SILUBRIN LUBRIFICAÇÃO INDUSTRIALLTDA. Ao final das $600 \mathrm{~h}$, o motor foi aberto e inspecionado visualmente, analisando-se as condições em que se encontrava.

\section{RESULTADOS E DISCUSSÃO}

Para representar o desempenho do motor ao longo das $600 \mathrm{~h}$ (cerca de 70 dias de ensaio), foram escolhidos os resultados referentes aos dias em que o motor completou intervalos de $100 \mathrm{~h}$. Com base nesses resultados, a variação da potência na TDP ao longo do período, cuja média ficou em torno de $38,1 \pm 0,6 \mathrm{~kW}$ (Figura 1), revela uma tendência de queda, enquanto que o consumo específico de combustível, cuja média ficou em $284 \pm 5 \mathrm{~g} \mathrm{kWh}^{-1}$, mostra comportamento crescente. Apesar disso, o uso da mistura B5 de sebo bovino ao diesel comercial B3 apresentou desempenho satisfatório do motor, que funcionou normalmente.

Ao comparar quatro combustíveis (Diesel puro, B2, B5 e B20 de sebo bovino) em motor diesel, GARCIA \& TOOKUNI (2006) observaram desempenho muito semelhante entre eles. Comparado ao diesel comercial, MAZIERO et al. (2009) observaram que o motor apresentou o mesmo desempenho com a mistura de biodiesel de sebo bovino e diesel comercial na proporção de $5 \%$.

Na tabela 1, os valores que mais chamam a atenção são os da viscosidade a $40^{\circ} \mathrm{C}$ e de insolúveis, que se mostraram superiores aos do óleo novo. Entretanto, os níveis de insolúveis (máximo 1,0\%), assim como a porcentagem de água (máximo $2,0 \%$ ), o número de basicidade total (TBN - mínimo 2,5mmKOHg min-1) mantiveram-se dentro de valores aceitáveis. De forma 

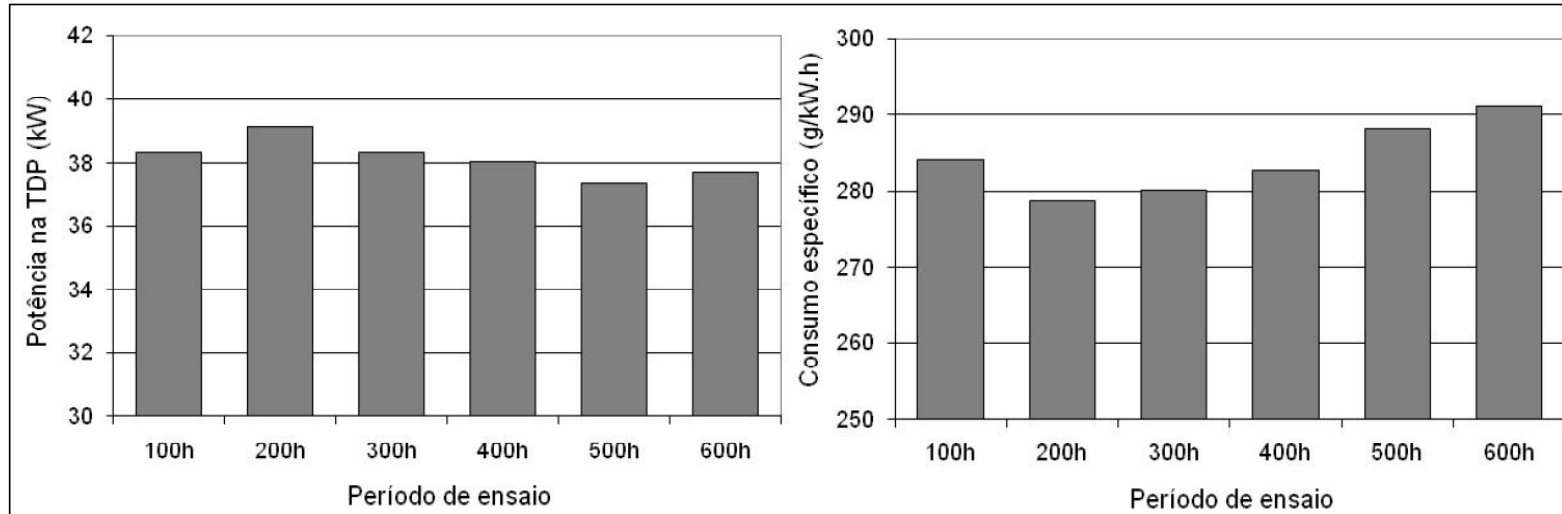

Figura 1 - Potência máxima da tomada de potência (Média: $38,1 \pm 0,6 \mathrm{~kW})$ e consumo específico à potência máxima $($ Média: $284 \pm 5 \mathrm{~g}$ $\mathrm{kWh}^{-1}$ ) ao longo das 600 horas de ensaio.

semelhante, INOUE et al. (2009) determinaram parâmetros físico-químicos de óleo lubrificante tais como, viscosidade a $40^{\circ} \mathrm{C}$, ponto de fulgor, TBN, água e insolúveis ao usar misturas de óleo de soja degomado ao diesel por 300h, não revelando discrepâncias ou alterações importantes.

Já os resultados de ensaios espectrométricos, que indicam e quantificam elementos químicos de desgaste metálico, contaminantes e aditivos, segundo laudos do Laboratório contratado para análise, apontaram alterações significativas em alguns componentes. Nas análises de 100 a 300h, a situação geral era de ALERTAe de 400 a 600h a situação geral era CRÍTICA. O parecer de cada um dos laudos segue abaixo: a) óleo novo: a análise da amostra indica que o lubrificante está em condições de uso, indicando um desempenho satisfatório do sistema; b) 100h: realizar cinco amostragens entre a troca periódica do lubrificante para o estabelecimento de tendências; 200h: alta concentração de $(\mathrm{Na})$, moderada concentração de (Fe), (Al) e (Si), aceitável concentração de $(\mathrm{Cu})$ e $(\mathrm{Cr})$; c) 300h: muito alta concentração de $(\mathrm{Na})$, alta concentração de $(\mathrm{Fe})$ e $(\mathrm{Si})$, moderada concentração de $(\mathrm{Pb})$ e (Al); d) 400h: alta concentração de (Fe), (Al), $(\mathrm{Si})$ e $(\mathrm{Na})$, moderada concentração de $(\mathrm{Cr})$, descontaminar o óleo com uso de equipamentos apropriados para remoção de partículas até a especificação desejada, inspecionar equipamento, filtros de óleo e de ar; e) 500 e 600h: alta concentração de (Fe), (Al), ( $\mathrm{Si})$ e (Na), descontaminar o óleo com uso de equipamentos apropriados para remoção de partículas até a especificação desejada, inspecionar equipamento, filtros de óleo e ar.

O nível de contaminação encontrado sugere que as trocas de óleo lubrificante devam ser a intervalos menores do que $200 \mathrm{~h}$ para evitar possível desgaste do motor.

A inspeção visual do motor no final dos ensaios é visualizada na figura 2, analisando-se as condições em que se encontrava. O que se pode constatar é que as pequenas deposições de carvão nos bicos injetores, cabeçotes e pistões (Figura 2A, 2B e 2C) são normais e compatíveis com as deposições que ocorrem com uso apenas de diesel, semelhantes às encontradas por INOUE et al. (2009).

Tabela 1 - Características físico-químicas do óleo lubrificante.

\begin{tabular}{|c|c|c|c|c|c|c|c|}
\hline Variáveis avaliadas & NOVO & $100 \mathrm{~h}$ & $200 \mathrm{~h}$ & $300 \mathrm{~h}$ & $400 \mathrm{~h}$ & $500 \mathrm{~h}$ & $600 \mathrm{~h}$ \\
\hline Aparência visual & LCC & LP & LP & LP & LP & LP & LCE \\
\hline Viscosidade a $40^{\circ} \mathrm{C}(\mathrm{cSt})$ & 99,1 & 100,4 & 121 & 111,5 & 108,4 & 110,6 & 128,6 \\
\hline Viscosidade a $100^{\circ} \mathrm{C}(\mathrm{cSt})$ & 15,1 & 16,4 & 16,4 & 17,53 & 16,9 & 16,4 & 19,6 \\
\hline Água por destilação (\%) & $<0,05$ & $<0,05$ & $<0,05$ & $<0,05$ & 0,05 & 0,05 & 0,05 \\
\hline Diluição $(\%)$ & 0 & 0 & 0 & 0 & Negat. & Negat. & Negat. \\
\hline Índice de basicidade TBN (mmgHOK $\left.\mathrm{g} \mathrm{min}{ }^{-1}\right)$ & 10,2 & 9,7 & 9,53 & 8,3 & 9,87 & 10,25 & 8,4 \\
\hline Ponto de fulgor $\left({ }^{\circ} \mathrm{C}\right)$ & 242 & 192 & 240 & 230 & 240 & 232 & 232 \\
\hline Insolúveis (\%) & 0,29 & 1,22 & 0,36 & 0,38 & 0,44 & 0,42 & 0,85 \\
\hline
\end{tabular}

Nota: $\mathrm{LCC}=$ Líquido Castanho Claro; $\mathrm{LP}=$ Líquido Preto; $\mathrm{LCE}=$ Líquido Castanho Escuro 


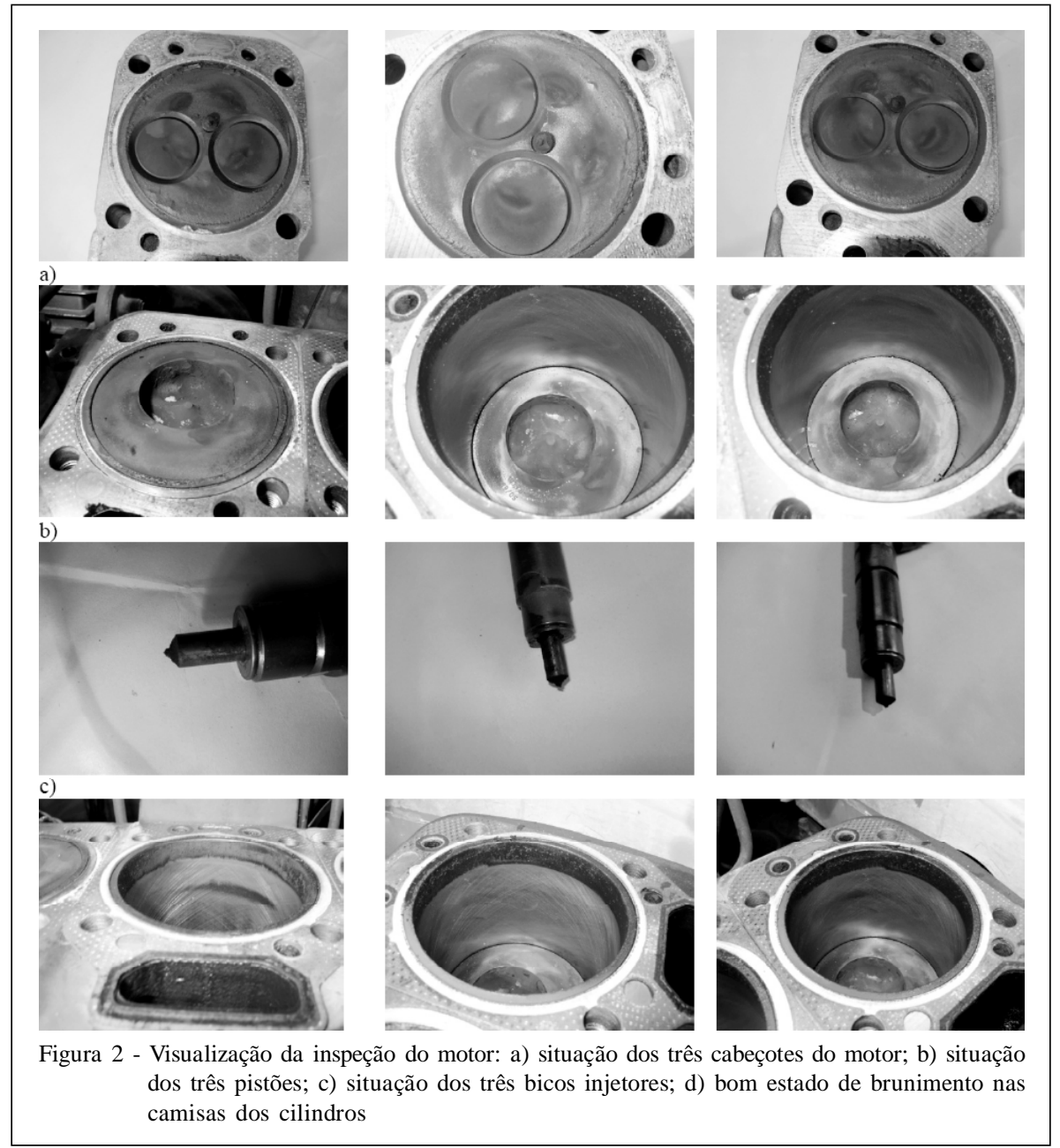

O estado de brunimento observado nas camisas dos cilindros (Figura 2D) também foi considerado normal, não caracterizando alterações significativas. O brunimentoé um processo de usinagem por abrasão após mandrilhamento, torneamento, fresamento, retificação interna etc., proporcionando uma geometria precisa do furo e bom acabamento superficial.

\section{CONCLUSÃO}

O uso de biodiesel de sebo bovino em mistura B5 mostra-se viável para uso prolongado em um motor diesel de injeção direta, havendo alterações no óleo lubrificante após $400 \mathrm{~h}$ de uso, sem, porém, causar danos aparentes aos componentes do motor.

\section{AGRADECIMENTOS}

À Fundação de Amparo á Pesquisa do Estado de São Paulo(FAPESP) (Proc. 2007/05005-0), pelo apoio financeiro à execução do projeto e ao Conselho Nacional de
Desenvolvimento Científico e Tecnológico (CNPq) (Proc. 472255/2007-0), pelo apoio financeiro à reforma do dinamômetro.

\section{REFERÊNCIAS}

ALI, Y.; HANNA, M.A. Durability testing of a diesel fuel, methyl tallowate, and ethanol blend in a Cummins N14-410 diesel engine. Transactions of the ASAE, St Joseph, v.39, n.3, p.793-797, 1996.

BETTO, T.L.; PRADO, E.A.P. Caracterização da estabilidade térmica de biodiesel proveniente de soja e de sebo bovino. In: ENCONTRO DE QUÍMICA DA REGIÃO SUL, 16., 2008, Blumenau,SC. Disponível em: <http://www.furb.br/temp_sbqsul/ _app/_FILE_RESUMO_CD/790.pdf $>$. On line. Acesso em: 29 ago. 2009.

BELLAVER, C. Nem só de vegetais vive o biodiesel. Revista Biodiesel, Monte Alto, v.26, p.16-17, 2008.

CORRÊA, I.M. et al. Desempenho de motor diesel com misturas de biodiesel de óleo de girassol. Ciência e Agrotecnologia, Lavras, v.32, n.3, p.923-928, 2008. 
GARCIA, A.J.M.; TOOKUNI, J.P.M. Biodiesel de gordura animal. Disponível em: <http://www.biodieselbr.com/estudos/ biodiesel/biodiesel-sebo-gordura-animal.htm >. On line. Acesso em 31 mar. 2010

INOUE, G.H. et al. Nota técnica: avaliação do desgaste de um motor de ciclo diesel alimentado com misturas de óleo vegetal e diesel. Engenharia na Agricultura, Viçosa, v.17, n.4, p.294302, 2009.

KAUFMAN, K.R.; ZIEJEWSKI, M. Sunflower methyl esters for direct injected diesel engines. Transaction of the ASAE, St. Joseph, v.42, n. 1, p.1626-1633, 1984.

MANSINI, Z.A.C., et al. O estudo da reação de transesterificação etílica do sebo bovino na obtenção de biodiesel, em diferentes condições de catálise. In: ENCONTRO REGIONAL DA SBQMG, 21. Uberlândia. 2007. Disponível em: <http://sec.sbq.org.br/ cdrom/31ra/resumos/T1551-1.pdf >. Acesso em 27 out. 2009

MAZIERO, J. V.G. et al. Desempenho de motor diesel com mistura de biodiesel de sebo bovino. Revista Biodiesel, Monte Alto, n.40, p.4-6, 2009. (Caderno Técnico).
MILLER KLEIN. Use of tallow in biodiesel, Out. 2006. Disponível em: <http://www.hgca.com/publications/ documents/Use_of_Tallow_in_Biodiesel.pdf $>$. On line. Acesso em: 2 dez. 2009.

MOURA, K.R.M. et al. Estabilidade térmica do sebo bovino e do biodiesel metílico e caracterização. Revista Biodiesel, Monte Alto, v.13, p.34-36, 2007.

PETERSON, C.L. et al. One-thousand-hour durability test with HySee and using a $5 \mathrm{X}$-Ema Test cycle. Transactions of the ASAE, St Joseph, v.42, n.1, p.23-30, 1999.

SALVADOR, N. et al. Viabilidade técnica do uso do éster metílico do óleo de Joannesia princeps, Vell. como combustível para motores diesel. Engenharia Agrícola, Botucatu, v.10, p.1423, 1986.

SILVA, M.V.I. da. Efeitos do uso do biodiesel sobre propriedades do óleo lubrificante usado em motor de ignição por compressão. 2006. 107f. Dissertação (Mestrado em Engenharia Mecânica) - UFSCAR/USP. São Carlos, SP. 